\title{
LE DIALOGUE SCOLAIRE, UN GENRE DISCURSIF FRONTALIER
}

\author{
Catherine Delarue-Breton
}

Université de Genève | «Raisons éducatives »

2019/1 N²3 | pages 47 à 69

ISSN 1375-4459

ISBN 9782940195930

Article disponible en ligne à l'adresse :

https://www.cairn.inforevue-raisons-educatives-2019-1-page-47.htm

Distribution électronique Cairn.info pour Université de Genève.

(C) Université de Genève. Tous droits réservés pour tous pays.

La reproduction ou représentation de cet article, notamment par photocopie, n'est autorisée que dans les limites des conditions générales d'utilisation du site ou, le cas échéant, des conditions générales de la licence souscrite par votre établissement. Toute autre reproduction ou représentation, en tout ou partie, sous quelque forme et de quelque manière que ce soit, est interdite sauf accord préalable et écrit de l'éditeur, en dehors des cas prévus par la législation en vigueur en France. Il est précisé que son stockage dans une base de données est également interdit. 


\title{
Le dialogue scolaire, un genre discursif frontalier
}

\author{
Catherine Delarue-Breton \\ Université de Rouen-Normandie
}

RÉSUMÉ - Trop souvent considérée comme une activité autosuffisante pour apprendre, permettant per se l'appropriation des savoirs, la verbalisation, qui recouvre des activités langagières variées, s'inscrit dans une forme dialogale qui va bien au-delà d'une simple conversation qui se trouverait avoir lieu à l'école. Situé à la frontière entre genre conversationnel et genre institué, le dialogue scolaire peut être considéré comme un genre discursif contraint par la sphère sociale dans laquelle il s'inscrit (l'école), et par les enjeux discursifs, pourtant opaques pour une partie des élèves, qui font sa raison d'être.

Cette contribution propose de définir les traits caractéristiques de ce genre discursif, et d'établir une typologie des significations produites par les élèves, utile pour mieux comprendre les inégalités d'apprentissage et de réussite scolaire. La description du dialogue scolaire et des significations qui s'y produisent conduira à définir quelques nécessités du discours pédagogique.

ABSTRACT - Verbalisation, which covers various language activities, is too often considered as a self-sufficient activity for learning, allowing the appropriation of knowledge. Indeed, it is part of a dialogical form that goes far beyond a simple conversation that would happen at school.

In between the conversational type and the established type of discourse, the school dialogue can be considered a discursive genre constrained by school - the social sphere in which it fits, and by the discursive issues, yet misunderstood by some students, which is at stake.

We will first define the characteristics of this discursive genre and to set up a typology of meanings produced by students. This is useful to better understand the inequalities of learning and of academic achievement.

Then, we will describe the school dialogue and the meanings that occur there.

This will lead to define some needs of the educational discourse. 
"D'un point de vue psychanalytique, le langage a pour vocation de faire partager un contenu d'affects, en relation avec une expérience du monde, et ce afin que l'ensemble puisse être élaboré par celui à qui le discours s'adresse, pour être ensuite restitué au sujet. » Danon-Boileau, 2005, p. 292.

\section{Introduction}

Danon-Boileau ne parle pas ici spécifiquement d'apprentissage ; pour autant, son propos nous parait emblématique du point de vue de l'apprenant, dans une relation d'apprentissage: l'expérience se développe et se transforme par le langage, dans l'interaction; l'un confie, l'autre élabore, puis restitue. Progressivement, l'élaboration est dévolue à l'apprenant... De ce point de vue, le langage n'est pas considéré comme le véhicule d'une pensée qui s'élabore en dehors d'elle, mais bien comme le lieu lui-même de l'élaboration de l'expérience.

Pour autant, si elle est nécessaire, l'élaboration de l'expérience par le langage n'est pas suffisante en soi pour produire les apprentissages scolaires, qui supposent une articulation entre expérience ordinaire du monde et disciplines scolaires, autrement dit une élaboration particulière, inscrite dans les catégories de pensée proposées au sein des disciplines scolaires.

S'intéresser aux rapports complexes entre langage et apprentissage consiste alors à regarder comment ou en quoi le langage, entendu ici comme usages de la langue en contexte scolaire, est partie prenante de l'apprentissage, et comment il permet ou non d'unifier l'expérience des élèves, scolaire et extrascolaire. À cet égard, nous retenons de la notion de verbalisation telle qu'elle est présentée dans l'introduction de ce numéro, d'une part sa fonction de cadrage de l'interlocution en contexte scolaire, d'autre part la conception qui la désigne comme activité du sujet en elle-même, et non comme moyen d'accéder à une autre activité. La verbalisation contribue ainsi aux processus de subjectivation.

Nous considérons cependant qu'entre usage spontané du langage et usage conscient, il existe des usages intermédiaires, qui puisent à des sources fort diverses, notamment du côté des élèves, usages ni tout à fait spontanés ni pleinement conscients, qui produisent des effets différents - et donc inégaux - en termes d'apprentissage. Le point de vue ici défendu est que toutes les formes discursives ne favorisent pas les apprentissages scolaires, notamment disciplinaires, et que certaines d'entre elles vont jusqu'à les défavoriser.

Nous proposons ici de faire le point sur les travaux que nous menons sur ce sujet depuis une douzaine d'années à partir de l'analyse d'un corpus constitué d'une cinquantaine de séances de classe du primaire enregistrées 
et transcrites, et d'en présenter une synthèse. Dans un premier temps, nous présenterons un modèle théorique d'analyse transdisciplinaire de ce que nous appelons le dialogue scolaire, qui met en avant les tensions parfois discrètes, mais actives néanmoins, auxquelles sont confrontés les élèves et les enseignants au cours des échanges langagiers scolaires, et qui sont susceptibles de contribuer à la production de malentendus. Nous décrirons ensuite les conditions de réussite de ce discours, et proposerons une typologie des significations produites par les élèves au cours des échanges, qui vise à mieux comprendre les différences de réussite et surtout d'apprentissage entre élèves d'une même classe. La question du rapport entre verbalisation et apprentissage ne peut cependant faire l'économie d'une réflexion sur le statut accordé au savoir en classe, et sur les conceptions de la langue et du langage qui l'accompagnent. Nous nous interrogerons donc également sur les conceptions du langage et des savoirs que semble implicitement véhiculer l'école française, et qui sont susceptibles de contrevenir au projet affiché par l'institution scolaire d'inscrire les élèves dans des démarches réflexives et problématisées.

\section{Le dialogue comme berceau des verbalisations : un espace discursif hétérogène}

Notre intérêt pour décrire les spécificités du dialogue scolaire a été suscité par le désir de mieux comprendre ce qui fait difficulté pour certains élèves dont le mode de socialisation familiale est moins en harmonie avec la socialisation scolaire que celui d'autres élèves. Un certain nombre de travaux ont montré en effet que les difficultés récurrentes de certains de ces élèves sont directement liées à ce qui se joue dans les échanges langagiers scolaires (voir notamment Bautier, 2008 ; Bautier \& Rochex, 2004) dans un contexte où le langage et la langue ont acquis, à l'école, une place prépondérante.

Si l'hétérogénéité constitutive des discours n'est plus à démontrer (Authier-Revuz, 1984), l'hétérogénéité spécifique du dialogue scolaire mérite d'être plus finement documentée. Les choix théoriques qui ont présidé à l'élaboration du modèle d'analyse que nous présentons ici relèvent d'une conception du dialogue entendue, à la suite de François (1990), comme espace discursif au sein duquel des significations se dessinent. Cette conception, qui se préoccupe de ce qui fait sens, se distingue de conceptions du langage au sein desquelles l'étude du sens est davantage centrée sur l'étude de la manière dont les énoncés (écrits ou oraux) sont compris par les élèves. C'est le processus même d'élaboration des significations que nous avons cherché à mieux comprendre et mieux décrire, et la manière dont s'élabore un système de significations, pour la classe et pour l'élève. Privilégier une approche des situations éducatives et des échanges en contexte scolaire en termes de production de significations au sein du dialogue (pertinentes ou non pertinentes), 
c'est considérer qu'au-delà d'énoncés objectifs qu'il s'agirait de comprendre, les élèves sont confrontés à des énoncés auxquels ils attribuent des valeurs et auxquels ils confèrent des significations hétérogènes, de portées très inégales pour s'approprier les catégories de pensée qui font le propre de l'enseignement scolaire (Delarue-Breton, 2016). Le recours à une conception psychanalytique du langage permet ainsi de prendre en compte la dimension des affects, qui ne sont pas à confondre avec les émotions, et qui jouent un rôle dans la compréhension du sens des activités pour les élèves ${ }^{1}$.

\section{La notion de dialogue}

Nous avons retenu, parmi un nombre conséquent de désignations possibles, le terme de dialogue pour caractériser les échanges langagiers entre élèves et entre maitre et élève(s) en classe pour plusieurs raisons; nous en présentons ici quelques-unes.

La première tient à la dimension non spécifiquement scolaire de la notion de dialogue; nous considérons en effet que le langage se développe tout autant dans des modes de socialisation, scolaires comme non scolaires, que dans des apprentissages formalisés ou explicites, ce que suggère davantage la notion de verbalisation. Or, ce sont précisément les usages sociaux, non formalisés du langage qui font que les élèves entrent à l'école dotés de dispositions inégales vis-à-vis des apprentissages impliquant le langage, autrement dit de dispositions inégales vis-à-vis des verbalisations proprement scolaires.

La seconde tient, à contrario, à la continuité que ce terme de dialogue permet d'établir avec la notion de cours dialogué et qui caractérise les échanges langagiers scolaires contemporains, favorisant plus ou moins, selon leurs modes de réalisation, l'enrôlement des élèves et la construction des savoirs, ou se réduisant à leur implication dans des tâches de bas niveau (Veyrunes \& Saury, 2009, p. 67).

La troisième enfin réside dans ce jeu constant des significations que le dialogue implique entre les interlocuteurs et l'objet de l'échange, qui est à la fois marge de manœuvre et source possible de malentendus : parce qu'activité conjointe ne signifie pas significations partagées (Bautier \& Rochex, 2004), le dialogue scolaire laisse place à des interprétations fort diverses, en termes d'enjeux notamment, et de ce fait constitue un espace discursif qui permet d'observer la manière dont se construisent et persistent les inégalités au sein même de l'école. C'est cet espace discursif que nous avons cherché à étudier dans sa dynamique, et non la production langagière des élèves en soi.

1. Nous ne développons pas ici cette distinction, mais signalons rapidement que dans cette acception, la notion d'affects suppose l'existence de représentations, certes en mouvement, mais émanant du monde intérieur de l'élève, et décrochées de la situation, tandis que les émotions sont considérées comme encapsulées dans le hic et nunc de la situation (Danon-Boileau, 1999). 
De ce point de vue, nos analyses ne disent rien de l'évolution des élèves : elles disent quelque chose de l'hétérogénéité des significations qui se dessinent au sein de la classe, dans le temps didactique.

Le dialogue constitue ainsi non seulement l'enveloppe verbale qui contient les significations, mais, en leur donnant forme, il contribue également à les produire ; loin d'être le véhicule ou le révélateur d'une pensée élaborée en dehors de lui, il est à la fois susceptible de dire quelque chose de l'activité psychique et intellectuelle des élèves, et de contribuer à la transformer.

\section{Le plan dialogal et le plan dialogique}

Au plan méthodologique, la progression de ce dialogue, constitué d'approximations successives (Danon-Boileau, 2006) visant simultanément à cerner l'objet concerné, et conduire les élèves à produire des significations pertinentes et stables permettant de l'intégrer à ce qu'à la suite de Bourdieu (1967/2007), nous désignons comme un corps commun de catégories de pensée, peut être étudiée sur deux plans (Bres, 2005):

- Sur le plan dialogal, à travers l'alternance des tours de paroles entre les interlocuteurs, dans le fil du propos, autrement dit dans sa continuité discursive.

- Sur le plan dialogique, à travers les effets d'échos que l'on peut observer entre des énoncés produits hic et nunc, dans le fil du propos, et des énoncés produits antérieurement et dans d'autres contextes.

L'intérêt méthodologique de ce double plan réside dans la possibilité qu'il offre de ne pas limiter l'étude des significations produites en classe à l'ici et maintenant du propos - un certain nombre de propos d'élèves, en effet, renvoie implicitement, voir inconsciemment, à des propos tenus en classe dans des séances antérieures - et à rétablir une forme de continuité, que nous nommons fil dialogique, dans la production de signification par les élèves, à travers des énoncés pourtant disjoints dans le temps.

Le corpus étudié, constitué d'une cinquantaine d'observations écologiques de séances de classe de $\mathrm{CP}^{2}$, a été enregistré (audio) et transcrit. Il se compose de vingt-huit séances de compréhension de dictons (corpus Delarue-Breton, 2012), de quinze séances d'apprentissage de la lecture (corpus Gomila, 2007), et de quelques séances de sciences. Bien que les séances de compréhension de dicton se soient déroulées à la suite les unes des autres, et sur une durée de presque cinq mois, nous ne parlons pas d'étude

2. $C P$ : Cours préparatoire, première classe de l'école élémentaire française, accueillant les élèves de 6-7 ans. Nous entendons par écologiques des séances où la présence du chercheur tend à être la moins influente possible. 
longitudinale, dans la mesure où nous n'avons suivi aucun élève de manière systématique. C'est le fonctionnement du dialogue scolaire dans son ensemble qui a été étudié, et qui nous a permis simultanément de repérer, au plan dialogal, des effets de rupture dans la continuité (quand le fil du dialogue se rompt, à la suite d'un propos incongru d'élève par exemple), et inversement, au plan dialogique, des effets de continuité à travers des énoncés disjoints, mais se faisant écho au plan linguistique. Nous en donnons un exemple ci-dessous (analysé dans Delarue-Breton, 2012).

[1] Ens. : qu'est-ce que ça veut dire que le $1^{\text {er }}$ avril on fait courir les sots $\lambda$

[2] Él. : ça veut dire que l'hiver souffle fort et que les sots s'en [vont] dans les pays chauds ${ }^{3}$

Le propos de cet élève, incongru, constitue une rupture dans le fil du discours, autrement dit une rupture dialogale. L'étude des séances antérieures conduit à établir un rapprochement avec celle qui a eu lieu quatre jours auparavant, et qui avait pour objet d'étude le dicton suivant : À la saint Gontran, si la température est belle, arrivent les premières hirondelles. Voici le propos qui conclut l'échange (nous soulignons) :

[3] Ens. : elles reviennent dans notre pays quand il fait bon donc elles viennent en fait des pays chauds hein voilà elles se sont envolées vers les pays chauds comme les pays d'Afrique du Nord par exemple hein voilà donc là elles reviennent chez nous puisqu'il va faire bon normalement à nouveau alors voilà pour le premier dicton

Le propos apparemment incongru de cet élève ne s'exempte donc en aucun cas du discours pédagogique : l'étude des phénomènes dialogiques contribue ainsi à rendre compte de ruptures dialogales.

\section{Entre dialogisme et polyphonie}

Toutefois, si à la suite de Bres (2005), nous parlons de dialogisme pour désigner l'articulation non maitrisée d'un énoncé hic et nunc avec un ou plusieurs autres énoncés, nous ne délaissons pas pour autant le terme de polyphonie, contrairement à certains auteurs (voir notamment sur ce point Bres \& Rosier, 2008), car les deux termes nous sont nécessaires.

Pour Bres (2005) en effet, la notion de dialogisme est préférable à celle de polyphonie, en ce qu'elle permet de prendre en compte la pluralité du sujet parlant; celui-ci fait observer que «parler de polyphonie [...] c'est penser la production de la parole en termes non de réponse mais de mise en scène

3. Passage extrait du corpus Delarue-Breton : Ens. désigne l'enseignante, Él. un élève, la flèche oblique [ $\ulcorner$ ] marque l'interpellation des élèves ou de l'un d'eux dans l'échange. 
énonciative » (p. 58), ce qui suppose un sujet «plein », dit-il, «maitre de sa parole comme de l'univers » (p. 58). À l'opposé, parler de dialogisme permet une approche en termes de réponses, donc en termes de production de sens plutôt qu'en termes de communication : «Au-delà des voix que le locuteur met en scène, il y a celles qu'il rencontre sans le vouloir, sans le savoir ; celles qui traversent son discours à son corps défendant [...]» (pp. 58-59).

En ce qui nous concerne, tout en privilégiant une approche responsive de l'étude du dialogue scolaire, nous employons le terme de dialogisme pour désigner les effets d'écho non maitrisés entre énoncés discontinus, et le terme de polyphonie pour désigner l'orchestration maitrisée par le locuteur des différentes voix qui traversent son propos. Cet usage des deux termes en complémentarité, et des conceptions sous-jacentes qu'ils recouvrent (sujet maitre vs non maitre de sa parole), permet de distinguer les usages verbaux spontanés de verbalisations plus formelles, tout en les inscrivant dans un continuum, un espace discursif non clivé, qui rend bien compte de l'hétérogénéité du sujet parlant. Le couple dialogisme/polyphonie permet ainsi de distinguer l'orchestration polyphonique des différentes voix qui constituent le dialogue scolaire - orchestration dont on peut considérer qu'elle est du ressort de l'enseignant, même si elle est parfois assumée par un ou plusieurs élèves - des effets de dialogisme non orchestrés, et susceptibles de contribuer au malentendu entre les locuteurs.

\section{Un genre discursif frontalier}

La notion de malentendu telle qu'elle se développe dans la sphère scolaire a fait l'objet d'un certain nombre d'études (notamment Halté, 1981 ; Altet, 1994 ; Bautier \& Rochex, 1997, pour citer les précurseurs), qui montrent la fragilité ou les failles de l'entente verbale entre maitre et élève(s), voire entre élèves en classe, et qui peuvent avoir des causes diverses.

Pour notre part, tout en nous inscrivant dans la suite de ces travaux, nous avons postulé que le malentendu était un phénomène inhérent au dialogue scolaire, pour des raisons tout autant structurelles que conjoncturelles. Nous avons ainsi tenté d'identifier les traits saillants de ce dialogue contraint par la sphère sociale dans laquelle il se déploie, entendu à la suite de Maingueneau (2004) comme genre discursif, constitué de l'intrication d'un texte (les échanges) et d'un lieu social (l'école), afin de chercher à comprendre dans quelle mesure ces malentendus récurrents relevaient - ou non - de l'accidentel. Ces travaux ont montré la dimension intrinsèquement frontalière de ce type de discours, tendu entre des dimensions contradictoires, susceptible de laisser place à des interprétations multiples, pas toutes également pertinentes pour apprendre; nous décrivons ci-dessous les tensions qui le constituent (pour plus de détail, voir Delarue-Breton, 2016, dont cet article constitue pour partie une synthèse). 


\section{À la frontière entre genre conversationnel et genre institué}

Dans sa description des genres discursifs, Maingueneau (2004) distingue deux grands régimes de généricités, les genres conversationnels, et les genres institués.

Les genres conversationnels se caractérisent par leur grande instabilité, liée aux stratégies d'ajustement et de négociations des interlocuteurs. Les genres institués, à l'opposé, revêtent une dimension non négociable de l'interaction verbale, aussi bien en termes de réception que de production et de distribution des rôles.

Cette définition linguistique du genre discursif, et plus particulièrement la bipartition entre les deux types de genres, nous semble particulièrement pertinente pour étudier le genre dialogue scolaire, dans la mesure où nous situons celui-ci précisément à la frontière entre genre conversationnel et genre institué. Cette première ambivalence, qui laisse pourtant place à l'unification de l'expérience des élèves, entre verbalisations formelles et moins formelles, contribue une source puissante de malentendus pour certains d'entre eux, dont le mode de socialisation familiale n'est pas en connivence avec les formes de socialisation scolaire (Bautier \& Rayou, 2009). Pour ces élèves en effet, la dimension conversationnelle des échanges en classe vient potentiellement masquer les enjeux discursifs de l'activité scolaire - et donc sa dimension instituée - et cette conversation demeure alors simple conversation à l'école, et non conversation scolaire. Le dialogue scolaire est ainsi conversationnel dans sa forme, ou dans ses modalités, mais institué dans ses enjeux discursifs : les échanges entre élèves, autour d'une figure géométrique par exemple, n'ont pas pour finalité l'échange lui-même, mais l'appropriation des propriétés disciplinaires de la figure en question.

Cette première tension entre genre conversationnel et genre institué, que nous décrivons comme caractéristique du dialogue scolaire, recèle cependant elle-même d'autres tensions, qu'elle vient en quelque sorte subsumer.

\section{Entre discours ${ }^{4}$ premier et second, discours horizontal et vertical, discours instructeur et régulateur}

Loin d'être homogène en effet, la dimension instituée de ce dialogue se caractérise par une hétérogénéité discursive que l’on peut analyser, elle aussi, comme tendue entre des pôles divergents.

4. Nous reprenons dans un souci de transparence le terme de discours (premier ou second, horizontal ou vertical, instructeur ou régulateur,) employé par ces différents auteurs; ce discours n'est cependant pas le même que le discours défini par Maingueneau, et que nous reprenons à notre compte. 


\section{Entre discours premier et discours second}

On doit à Bakhtine (1979/1984) l'opposition entre discours premiers et discours seconds, et à Schneuwly (1998), Jaubert \& Rebière (2002), Bautier \& Rochex (2004), et d'autres encore, son transfert dans la sphère scolaire. Ces travaux ont montré notamment en quoi l'activité langagière, entendue comme partie prenante de l'apprentissage, pouvait se définir comme une activité de secondarisation, donc de ressaisie et de reconfiguration de discours premiers, le discours second n'étant pas une fin en soi, mais le nouveau point de départ d'un processus indéfiniment réitéré de reconfiguration des significations. Le périmètre dans lequel s'inscrit cette activité de secondarisation fait cependant l'objet de controverses entre chercheurs, qui considèrent soit que celui-ci s'inscrit tout entier au sein des disciplines, soit qu'il les déborde.

De notre point de vue, si la secondarisation s'inscrit dans des processus d'élaboration de soi qui vont certes au-delà de l'inscription dans des communautés discursives disciplinaires, il y a aussi lieu d'envisager, dans la définition scolaire de la secondarisation, son rapport étroit avec les principes de classification des disciplines. Secondariser à l'école signifie ainsi pour nous ressaisir un objet mondain (ou objet de l'expérience ordinaire) pour le référer - même implicitement - à une catégorie en lien avec les champs disciplinaires enseignés à l'école. Parler de droite en mathématiques, d'espace en géographie ou de récit en français, pour désigner un ensemble de propriétés théoriques, se réfère à ces principes de classification, ce qui confère à la secondarisation le statut de nécessité scolaire traversant les disciplines, mais inscrite cependant dans chacune d'entre elles. Or, nous considérons que ce que vient masquer la dimension conversationnelle du dialogue scolaire, pour certains élèves, c'est précisément l'exigence de secondarisation de l'activité discursive, qui fait sa raison d'être. Mais comment cette dimension conversationnelle en vient-elle à masquer la visée discursive des échanges ? Le recours à la notion de discours vertical/horizontal proposée par Bernstein (2007) nous permet de répondre en partie à cette interrogation.

\section{Entre discours horizontal et discours vertical}

Pour le dire brièvement, Bernstein définit le discours horizontal comme un discours segmenté et contextualisé, où les catégories sont implicites, tandis que le discours vertical se caractérise par une structure cohérente et explicite. Or, les échanges dialogués en classe participent selon nous de l'un et de l'autre, se situent donc à la frontière entre verticalité et horizontalité, dans des proportions variables selon les contextes, que ce soit en fonction de l'école, en fonction de la classe, ou en fonction de l'enseignant, mais au sein d'une même classe, nous considérons qu'il existe également des différences en fonction du type d'activité, du type d'élève concerné etc. Cette opposition nous permet de mieux comprendre les circuits par lesquels les significations sont susceptibles de se dessiner, et notamment, de comprendre que le discours pédagogique, qui comporte entre autres les éléments de contenu, n'est pas entièrement inscrit dans le discours enseignant: le texte de savoir se 
développe aussi à travers les propos des élèves, ce qui pose la question de sa restitution au collectif que représente la classe. En quoi consistent, cependant, ces contenus?

\section{Entre discours instructeur et discours régulateur}

Pour Bernstein (2007), le discours pédagogique se définit comme l'imbrication de deux autres discours, le discours instructeur et le discours régulateur. Le discours régulateur renvoie aux conditions de régulation de l'activité, tandis que le discours instructeur renvoie aux contenus de savoirs. Nous précisons toutefois que les types de savoirs scolaires auxquels nous nous intéressons ici sont les savoirs que l'on peut dire savants ou théoriques, autrement dit des savoirs conceptuels (nous avons évoqué précédemment les notions de droite, d'espace ou de récit par exemple). D'autres types de savoirs sont certes enseignés à l'école, mais ceux que nous prenons en compte dans notre approche sont les savoirs théoriques, car ce sont ceux qui présentent le plus d'altérité pour les élèves qui doivent se les approprier.

Bien que les éléments régulateurs, qui visent davantage l'effectuation de la tâche que l'explicitation de ses enjeux classificatoires, l'emportent généralement, selon Bernstein, dans le discours pédagogique, nous avons pu observer que certains élèves, dont le mode de socialisation familiale est le plus connivent de la socialisation scolaire, privilégient pourtant de manière récurrente les éléments instructeurs, aussi discrets ou implicites soient-ils (Delarue-Breton, 2012). Ce constat n'est pas anodin : c'est sans doute cette perception claire, par certains élèves, des enjeux discursifs disciplinaires du dialogue scolaire, fussent-ils implicites, qui empêche les enseignants de comprendre ce qui peut faire difficulté pour les autres. Mais comment se déploient, dans la progression des échanges, ces éléments instructeurs?

\section{Des temps d'approximations successives et des temps de stabilisation du savoir}

Si certains travaux ont montré le caractère ternaire des échanges en classe, de type initiation/réponse/évaluation (Sinclair \& Coulthard, 1975), le recours aux notions de jugements d'approximation et jugements par catégorisation stricte travaillées par Danon-Boileau (2006) permet de mettre au jour une autre forme de structuration récursive du dialogue scolaire, à deux temps, consistant pour l'un en approximations successives visant à cerner l'objet de savoir, et pour l'autre en affirmations de l'ordre de la catégorisation stricte permettant sa stabilisation. Nous considérons en effet que s'approprier les propriétés d'un objet conceptuel en classe implique une sorte de rumination ${ }^{5}$

5. Nous remercions affectueusement ici Irène Fenoglio, à qui nous empruntons ce terme de rumination, qu'elle a employé en notre présence dans un autre contexte, mais toujours pour parler de rumination théorique... 
collective de l'objet par le sujet, qui l'élabore activement dans l'échange (et non qui s'assujettit à l'objet), autrement dit qui le rapporte à son expérience et le situe par rapport à celle-ci, et ce faisant reconfigure les connaissances qu'elle a pu contribuer à produire. Cette rumination se déploie en classe de manière itérative, et se voit jalonnée par des temps de stabilisation, généralement sous forme assertive.

En voici un exemple ${ }^{6}$ :

[1] Lauren entoure

[2] E entoure qu'est-ce que ça veut dire $\lambda$

[3] Lauren on entoure on entoure un mot

[4] E entoure un mot

[5] Margot parce que c'est entoure un petit mot dans l'histoire de Ratus

[6] E

[7] Franck entoure un objet

[8] E entoure un objet est-ce que là dans l'histoire Ratus entoure un objet $\lambda$

[9] LL non

[10] E est-ce qu'il entoure un mot $\nearrow$

[11] LL non

[12] E alors qu'est-ce qui entoure Ratus $\nearrow$

[13] Manon les gens les gens les gens entourent Ratus

[14] E les gens entourent Ratus ça veut dire quoi les gens entourent Ratus $\pi$ Stanislas ça veut dire quoi les gens entourent Ratus $\lambda$

[15] Stanislas ils l'encerclent

[16] E ils l'encerclent bien ça veut dire quoi encore Clémence $\lambda$

[17] Clémence ça veut dire qu'ils sont autour de lui

[18] E tout autour de lui très bien ils l'entourent ils sont tout autour de lui changez de mot Manon $\nearrow$

On peut observer dans ce court passage des temps d'approximation de l'objet visé, qui porte sur différentes acceptions du verbe entourer, constitués par exemple des tours de parole [2], [3], [4], [5], où on voit Margot faire appel à l'expérience commune (" entourer un petit mot dans Ratus»), ou encore des tours [8] à [14], où l'enseignante réoriente l'échange vers l'acception être autour de. On observe tout autant la présence de temps de stabilisation, comme dans le tour de parole [6], où l'enseignante autorise le rapprochement avec l'expression «entourer un mot » (« oui, c'est pareil »), et valide provisoirement l'acception mettre autour de, ou encore le tour de parole [16], où

6. Ce passage est extrait du corpus Gomila (2007), et vise l'appropriation par les élèves de certains termes; il s'agit en l'occurrence du verbe entourer. E désigne l'enseignante, $L L$ plusieurs élèves qui parlent en même temps. 
l'enseignante valide le synonyme encercler, qui marque le changement d'acception (encercler = être autour de) en répétant la proposition ( «ils l'encerclent »), et en la commentant («bien »), et enfin le tour de parole [18], qui valide la périphrase tout autour de lui («très bien »), clos l'échange et arrête définitivement la quête autour de ce terme («changez de mot »). Cet exemple illustre bien la bipolarité du dialogue scolaire, mais aussi le caractère relativement aléatoire, voire imbriqué des temps d'approximation et de stabilisation du savoir, qui alternent sans régularité, en fonction des apports des uns et des autres notamment, que Maingueneau appelle stratégies d'ajustement et de négociation des interlocuteurs. Dès lors, on peut rendre compte de certaines difficultés d'élèves selon deux points de vue, qui peuvent éventuellement se combiner:

- L'absence ou la fragilité de l'équilibre entre approximations successives et stabilisation du savoir (on observe en effet des formes de progression du dialogue scolaire où les approximations successives, se réitérant sans cesse, ne sont pas ou très peu arrêtées par des temps de stabilisation du savoir; d'autres au contraire où les propriétés des savoirs concernés sont assertées sans autre forme de procès, au détriment des temps d'approximation).

- La non-implication, pour des raisons diverses, de certains sujets dans l'échange collectif, et leur non-perception des visées de ces différents temps.

Ainsi, la verbalisation scolaire, dans ses dimensions instituées, formelles au sens de formalisées à des fins d'apprentissage, se déploie selon deux modalités qui ne peuvent se substituer l'une à l'autre : élaboration du savoir d'une part, stabilisation du savoir d'autre part, impliquant quant à elle des jugements par catégorisation stricte, qui ne souffrent pas l'approximation. La difficulté, pour l'enseignant comme pour l'apprenant, de ce type de discours, est de tenir ensemble des modalités discursives qui reposent en quelque sorte sur deux conceptions du langage. La première, qui s'inscrit dans la tradition de l'aristotélisme, privilégie l'exactitude, la classification en traits nécessaires et suffisants, qui implique le jugement par catégorisation stricte, et ne laisse pas place à l'élaboration verbale; elle suggère l'existence d'une forme de coïncidence entre les mots et les choses, et donc l'idée d'une certaine transparence du langage. L'autre, qui privilégie le tâtonnement, donc l'approximation dans son acception la plus étymologique, s'inscrit dans une tradition plus wittgensteinienne, où les objets font l'objet de catégorisations qui empruntent à la comparaison, à l'analogie, au rapprochement dans toutes ses formes, bref, qui reposent sur un « air de famille »; nous reviendrons plus loin sur ces conceptions, et la nécessité de les tenir ensemble.

Pour résumer notre propos, si ces échanges dialogués s'inscrivent bien dans le genre discursif frontalier que nous avons décrit précédemment, qui 
offre intrinsèquement un espace propice au développement de malentendus, ce genre est cependant susceptible de s'actualiser en scénographies fort différentes. Les malentendus sont donc tout autant le fait de la scénographie retenue que du genre discursif dialogue scolaire.

Les unes relèvent en effet du jeu de questions-réponses fermé, où la parole de l'élève est fortement circonscrite, d'autres, au contraire, du dialogue conversationnel largement ouvert, peu distinct de fait des dialogues non scolaires ; d'autres, enfin, s'inscrivent dans un dialogue articulant plus favorablement temps d'approximation et temps de stabilisation du savoir, favorisant ainsi l'élaboration de significations pertinentes pour apprendre, c'est-à-dire susceptibles de s'intégrer dans un corps commun de catégories de pensée en lien avec les disciplines étudiées, et permettant l'élaboration conjointe de soi et du monde. Sans qu'il soit possible de définir de manière ferme et immuable ce qu'est une scénographie pertinente, il est cependant possible d'imaginer l'existence d'une corrélation entre type de scénographie et type de signification produite par l'ensemble des élèves.

\section{Des significations différenciées}

Pour Maingueneau (1998), les conditions de réussite d'un genre se définissent à travers sa finalité, son organisation en tant que texte, ses conditions temporelles et spatiales, le rôle des énonciateurs, et le rôle des supports. Du point de vue de sa finalité, que nous préférons formuler en termes d'enjeux, au pluriel, on peut s'interroger aussi bien sur la manière dont l'institution scolaire les définit, que sur la manière dont les acteurs ou les usagers du système éducatif les perçoivent ou se les représentent. L'étude présentée ici a permis de mettre en évidence l'hétérogénéité de ces représentations parmi les élèves, à partir de l'analyse des significations produites au cours des échanges en classe.

Sans aborder ici le redoutable problème théorique que représente encore la notion de signification pour le linguiste, signalons simplement que l'emploi du terme signification dans nos travaux vise, dans la tradition benvenistienne, à privilégier l'étude du sens que les élèves confèrent aux énoncés, aux activités, ou aux objets auxquels ils sont confrontés, et non à regarder seulement ce qu'ils comprennent du sens inscrit dans ces énoncés, activités, objets etc. Dans cette acception, il s'agit toujours de comprendre comment ils peuvent «faire sens » avec cela ; autrement dit, le terme désigne aussi bien les significations qui se déploient dans le dialogue, et celles que les élèves confèrent aux situations qui leur sont proposées, qui sont généralement imbriquées et difficilement séparables. Dès lors, la notion de signification, qui n'est pas vraiment distinguable de celle de sens chez Benveniste (Normand, 1996), nous permet d'élaborer une première typologie visant à distinguer des manières différentes de conférer du sens à des énoncés, des activités, des objets etc., qui se manifestent dans les propos des élèves. 
L'étude des données précédemment mentionnées a ainsi permis de distinguer un triple idéaltype permettant de caractériser les significations produites par les élèves au cours des échanges: des significations que nous avons qualifiées de mondaines, des significations que nous avons qualifiées de formelles, et des significations que nous avons qualifiées de culturelles.

\section{Des significations mondaines, formelles et culturelles}

Charlot, Bautier, et Rochex (1992) avaient déjà proposé un idéaltype permettant d'opposer des positionnements d'élèves inscrits dans un rapport plus immédiat au monde, centré sur l'activité elle-même, à des positionnements inverses, visant, au-delà des activités, l'appropriation des principes généraux des domaines ou disciplines concernés. Cet idéaltype, qui permettait d'analyser des entretiens conduits avec des élèves, ne rend cependant pas compte de positionnements que l'on rencontre en situation écologique, caractérisés par des formes verbales conformes aux attendus au sein de la classe, ou qui reprennent à l'identique des formulations du discours pédagogique, sans qu'il soit possible de distinguer si l'élève s'est approprié la seule enveloppe verbale des savoirs concernés, ou réellement leurs propriétés ; or, ce type de positionnement est au moins aussi fréquent que les deux autres. Le modèle d'analyse que nous proposons vise ainsi à intégrer ce niveau formel des significations, que nous avons appelé ainsi car les propos des élèves dans ce cas reprennent la forme (verbale) des énoncés, et ne reflètent pas nécessairement l'appropriation des propriétés qu'elles recouvrent.

La notion de significations mondaines permet de caractériser des énoncés au sein desquels la part de ressaisie des objets du monde est peu importante, qui s'inscrivent dans un échange qui se distingue peu d'une conversation ordinaire, mondaine au sens où elle convoque des objets et des fonctionnements du monde plutôt que des objets et des fonctionnements du savoir. De ce point de vue, les indicateurs qui permettent de qualifier les significations ne sont donc pas tant les mots eux-mêmes que l'usage qui en est fait, en lien avec l'activité de l'élève ; autrement dit, ce sont des indicateurs discursifs plutôt que linguistiques.

Ainsi, on peut parler de significations mondaines quand il est demandé aux élèves de colorier les différentes parties d'une plante et de son environnement, en attribuant une couleur par type d'élément (par exemple une couleur pour les racines, une autre pour la tige, une autre pour les feuilles, une autre pour la terre, etc.), et qu'un élève choisit ses couleurs en fonction de son expérience du monde, par exemple vert pour la tige, les feuilles, etc. Dans ce cas de figure, l'élève privilégie, dans l'organisation des couleurs choisies, la dimension du monde, à la dimension symbolique que visait pourtant l'activité. Il ne s'agit pas ici d'affirmer que cet élève n'apprend pas, mais de repérer qu'il ne ressaisit pas la notion de couleur, considérée dans l'expérience ordinaire 
comme une qualité visible de la matière, mais qui se rapporte dans l'exercice proposé à un code symbolique, défini en fonction des propriétés ou caractéristiques de la plante. Au plan langagier, la notion de signification permet ainsi de saisir ce que le discours signifie, ce qu'il dit de l'expérience en train de se vivre, au-delà des mots eux-mêmes.

On pourrait être tenté de considérer que ces significations mondaines sont le produit de scénographies exemptes de verbalisations explicites, visant à formaliser les savoirs scolaires, et ainsi empêcher la suprématie des significations du monde ; pourtant, certaines verbalisations, parce qu'elles se focalisent sur l'enveloppe verbale des propriétés des savoirs, nous sont apparues tout aussi contreproductives.

La notion de significations formelles permet de caractériser, nous l'avons dit, des énoncés qui s'évertuent à prendre en compte et restituer dans le dialogue scolaire les attentes verbales ou attentes verbales supposées (termes, tournures syntaxiques, etc.) déjà proposées ou validées par l'enseignant, sans que l'on puisse s'assurer, à travers la seule analyse du discours, que l'élève s'est effectivement approprié les propriétés attendues. Ainsi, quand un élève rappelle les marques typographiques d'une liste "un titre, des mots en colonne, des tirets », en reprenant les mots exacts de l'enseignante, mais qu'il ne sait pas reconnaitre une liste qu'il n'a pas déjà vue, on peut considérer qu'il a appris une formule, et non appris à identifier ou utiliser les propriétés d'un objet de savoir.

La notion de significations culturelles renvoie à une élaboration des savoirs personnelle et singulière (nous entendons par là une élaboration intrasubjective, qui s'est cependant construite dans le collectif, dans des relations intersubjectives) et inscrite dans la durée. Nous empruntons le terme à Winnicott (1971/1975), qui définit l'expérience culturelle comme une activité indéfiniment réitérée de nouage des significations internes et externes au sujet. Ce type de significations se repère aisément, dans la mesure où l'élève qui les produit en vient souvent à mentionner des éléments nouveaux et pertinents concernant les caractéristiques d'un objet de savoir, que l'on ne retrouve pas dans le discours pédagogique. Ainsi, pour reprendre l'exemple précédent, concernant l'objet liste, lorsqu'un élève indique que dans certaines listes il y a des chiffres et non des tirets, élément qui n'est pas apparu dans le cours, on peut considérer qu'il s'est approprié l'objet, au-delà de son enveloppe verbale.

Rappelons cependant que cet idéaltype vise à analyser des discours, et propose une typologie des significations produites en leur sein, et non les personnes qui les produisent. Ainsi, on peut rencontrer tout type de signification dans les propos d'un élève, sans en déduire qu'il s'inscrit ou ne s'inscrit pas dans l'expérience culturelle. C'est la récurrence, voire la prépondérance d'un type de significations au sein des discours produits par un même élève sur la durée et dans des contextes différents qui pourrait alerter sur le malentendu dans lequel il s'inscrit potentiellement. 
Enfin, indiquons que l'intérêt de cette catégorisation réside selon nous dans la mise au jour des significations formelles, qui peuvent traduire une attitude - réversible, on le dit à nouveau - d'assujettissement ou de complaisance vis-à-vis des savoirs, consistant en l'introjection dans la psyché de significations "toutes faites », et non une attitude créative, permettant l'appropriation et l'utilisation de ces savoirs dans des situations inédites (Delarue-Breton, 2016). Or, nous nous interrogeons sur le fait que cette attitude puisse être implicitement encouragée par l'école.

\section{Une conception (trop) aristotélicienne de la langue?}

Bronckart (1987) rappelle le fait que la langue ne procède pas d'un rapport direct entre le mot et la chose (Authier-Revuz, 1984, parle elle-même de non-coïncidence des mots et des choses), et souligne que les significations langagières procèdent d'un rapport à l'extralangagier : « L'usage est le produit de l'action » dit-il, et « l'usage verbal est plus particulièrement le produit d'interactions langagières » (p. 30); et plus loin : «La signification, c'est le produit de l'usage verbal » (p. 45). Or, ce qu'il désigne comme « aristotélisme linguistique », entendu comme conception du langage considéré comme transparent, où « les mots ont un sens - et un seul - que chacun peut reconnaitre » (p. 30), semble difficile à dépasser. Plus de trente ans après ce constat, il nous apparait que l'école, notamment en France, qui promeut pourtant la réflexivité dans toutes ses dimensions, demeure pour partie prisonnière de cette conception aristotélicienne de la langue.

Au plan historique, Authier-Revuz (2003) rappelle que le caractère autonymique de la langue (quand un terme peut désigner un référent objectif, ou le terme même qui désigne ce référent), qu'elle considère comme pièce maitresse de sa réflexivité naturelle, a été considéré de deux manières opposées dans l'Histoire. Pour les uns, dans la tradition d'Aristote, et des logiciens qui ont suivi, jusqu'à aujourd'hui, cette réflexivité, et particulièrement l'autonymie, est

[...] un défaut des langues naturelles, venant, par des confusions entre usage et mention, perturber la mécanique logique du calcul du vrai (par les paradoxes produits). Devant ce danger, la question est avant tout d'en limiter au maximum les dégâts, avec une certaine fétichisation pour les dispositifs de notation désambigüisatrice comme le guillemet. (p. 67)

Pour d'autres au contraire (Benveniste, Jakobson, Authier-Revuz notamment), dans la suite desquels nous nous inscrivons, la réflexivité est une propriété spécifique des langues naturelles, considérée comme positive, puisqu'elle permet à la langue de parler d'elle-même (Authier-Revuz, 2003, pp. 69-70). Or, la récurrence, dans les prescriptions institutionnelles, de formulations qui invitent les enseignants à développer chez les élèves un rapport au langage 
exact, correct (on note par exemple dans le programme de $2008^{7}$ [Ministère de l'Éducation nationale, 2008] huit occurrences d'emploi du terme exact ou de l'un de ses dérivés, et trente occurrences d'emploi du terme correct ou de l'un de ses dérivés pour désigner l'usage de la langue), nous semble précisément traduire une inclination marquée pour les situations univoques ou désambigüisatrices, signalées par Authier-Revuz. Pour autant, cette mise en avant de l'univoque dans la langue est susceptible de détourner certains élèves d'une posture labile, nécessaire pour développer des compétences permettant de s'exercer au jeu des choix langagiers. Il ne s'agit bien évidemment pas de contester la nécessité de développer chez les élèves la possibilité de s'exprimer dans le respect des normes structurelles de la langue, ou de disposer d'un vocabulaire varié permettant une expression précise et juste. C'est d'ailleurs pour cette raison que nous suggérons dans le titre de cette section la possibilité d'une conception (trop ?) aristotélicienne de la langue : un peu d'aristotélisme, si l'on peut dire, ne nuit pas. Mais l'obsession de l'univoque, parce qu'elle rend invisible cette dimension toujours possible du choix (le mot juste étant précisément celui qui a fait l'objet d'un jugement), risque d'entretenir, chez les élèves pour lesquels ce rapport labile au langage n'est pas instauré par la socialisation familiale, un rapport figé et non créatif (un rapport complaisant dirait Winnicott) au langage, mais aussi au savoir et au monde.

\section{Verbalisation et problématisation}

Car une telle conception du langage coïncide avec une conception du savoir également figée, qui nous semble contrevenir sérieusement aux ambitions, affirmée par l'école, d'engager tous les élèves sur la voie de la problématisation. Fabre (2007) est l'un des premiers à avoir montré les risques, pour l'école, de ce qu'il nomme une réification des savoirs, qui affecte les théories de la connaissance depuis l'Antiquité et qui présente les savoirs indépendamment des questions qui les ont suscités:

Tel est donc le mécanisme du refoulement problématologique, ou encore de la chosification, qui se met en place dès l'origine de la philosophie occidentale et qui affectera toutes les théories de la connaissance de la modernité [...]. Ses conséquences sont immenses pour le savoir en général, et pour le savoir scolaire en particulier. Tout savoir constitue bien une réponse, mais cette réponse, une fois obtenue, refoule la question à laquelle elle répond en occultant du même coup son caractère de réponse. Elle parait alors valoir en soi. (Fabre, 2007, pp. 71-72)

7. Le programme de 2015 (Ministère de l'Éducation nationale, 2015) ne contient plus ces occurrences ; la présence massive (plus de 70 occurrences) du terme expliciter (ou de l'un de ses dérivés) semble cependant faire également écho à une nécessité de transparence du langage. 
Présenter les savoirs indépendamment des questions qui les ont suscités, et donc comme des savoirs isolés, ou valant en soi, en fait des savoirs dé-théorisés, donc des savoirs formels, qui ne s'inscrivent pas dans un corps de savoir (la théorie), mais demeurent juxtaposés, et font figure de fin en soi et non de concepts utiles ensemble pour penser et comprendre le monde. Le caractère déconnecté en classe des réponses et des questions qui les ont suscitées en vient ainsi à dévitaliser les savoirs convoqués, et, au-delà, à faire obstacle à l'unification de l'expérience des élèves. À l'opposé, le statut d'un savoir envisagé dans ses liens possibles avec l'expérience et les questionnements propres des élèves, permet de le penser comme susceptible de restaurer un continuum provisoire mais nécessaire entre théorie et expérience personnelle, et dès lors comme pivot de l'unification de l'expérience, qui nous semble être précisément un des domaines où se creusent les écarts entre élèves (Delarue-Breton, 2016).

Or, une conception du savoir qui promeut sa dimension responsive au détriment de la dialectique entre questionnement et réponses implique des formalisations langagières de ce savoir particulières, qui font écho à une conception propositionnelle de la pensée (Fabre, 2007). Dans ce cas, le savoir (comme proposition logique) est réduit à des assertions qui mettent en avant leurs contenus propositionnels, c'est-à-dire ce qu'elles disent sur le monde, comme des dires autonomes (Fabre, 2007). Autrement dit, les savoirs formalisés sont présentés comme des propositions logiques, qui valent en tant que telles, ce qui est préjudiciable aux élèves, et plus particulièrement à certains d'entre eux. Il nous semble dès lors possible de rapprocher conception propositionnaliste du savoir et conception aristotélicienne de la langue, susceptibles d'entrainer chez les élèves une conception illusoirement transparente de la langue à l'école, et dans le même temps une conception formelle voire absolue du savoir. Ce rapprochement permet de comprendre en quoi l'école est susceptible de contribuer à l'accroissement des inégalités : statut du langage et statut du savoir semblent parfois y valoir en soi, dans un figement contreproductif pour accompagner le développement d'un corps commun de catégories de pensée, qui ne peut exister que dans une conception souple et dynamique du savoir et du langage, où les catégories sont simultanément fermes et évolutives, et les savoirs, des certitudes provisoires, pour reprendre la formule de Fabre (Delarue-Breton, 2016).

\section{Conclusion : quelles nécessités du discours enseignant?}

Étudier le rôle, les modalités, les effets de la verbalisation en classe sur les apprentissages des élèves revient pour nous à chercher à comprendre «comment, à certaines conditions [...] les productions intersubjectives (le discours produit au sein de la classe entre ses différents acteurs) deviennent des 
ressources intrasubjectives, donc des possibles langagiers et linguistiques, ce faisant cognitifs » (Bautier, 2008, p. 134).

Prendre en charge un questionnement de ce type a consisté d'abord pour nous à nous interroger sur les traits caractéristiques du dialogue scolaire, et à chercher à mieux comprendre les fonctionnements discursifs complexes de ces échanges langagiers socialement situés, afin de mettre en évidence les formes d'hétérogénéité, zones d'opacité et tensions diverses qui leur sont inhérentes.

Nous avons retenu la notion de dialogue comme berceau des verbalisations scolaires - terme que nous réservons à la part formalisée des échanges en classe - afin de ne pas négliger la prise en compte de ce qui peut faire sens pour les élèves, au-delà du sens inscrit dans les énoncés. Ce dialogue, situé en tant que genre discursif à la frontière entre conversationnel et institué, est constitué en outre de tensions à différents niveaux : entre discours premier et second, entre discours horizontal et vertical, entre discours instructeur et régulateur notamment. II ne s'agit cependant en aucun cas de dénoncer ces espaces ambivalents, qui représentent les marges de manœuvre du locuteur, en l'occurrence du locuteur scolaire, et donc les marges de manœuvre de l'apprenant, qui évolue par approximations successives. C'est en effet en cheminant, par exemple en géographie, des plis de la jupe (objet du monde) au plissement de terrain (objet de savoir), ou en mathématiques du trait (objet du monde) à la droite (objet de savoir), ou encore en français du théâtre comme lieu (objet du monde) au théâtre comme genre littéraire (objet de savoir) que les élèves peuvent s'approprier le corps commun de catégories que constituent les disciplines scolaires. Ces approximations successives visant à cerner progressivement l'objet de savoir, afin d'en approcher les propriétés, doivent cependant être arrêtées par des énoncés destinés à les formaliser, contribuant ainsi à leur stabilisation, au moins provisoire. C'est à cette condition en effet que la confrontation intersubjective des points de vue dans les échanges en classe peut être à même de contribuer à l'appropriation intrasubjective de ces objets. L'étude des significations produites au sein de ce dialogue scolaire par les uns et les autres montre en effet qu'elles ne sont pas toutes de même nature, et inégalement pertinentes pour apprendre : si les significations que nous avons qualifiées de culturelles attestent d'une appropriation des objets de savoir visés, celles que nous avons qualifiées de mondaines traduisent une indistinction entre objet du monde et objet de savoir, et celles que nous avons qualifiées de formelles, d'une appropriation de l'enveloppe verbale des propriétés, mais pas nécessairement des propriétés elles-mêmes.

L'étude des spécificités discursives de ce dialogue, et des significations qui s'y produisent, permet donc de déduire des nécessités du discours enseignant, dont le rôle, au sein des pédagogies de l'activité, n'est pas d'évidence. Nous ne prétendons évidemment pas répondre de manière définitive à cette question, mais souhaitons mettre en avant quelques principes, susceptibles 
de contribuer à intégrer dans le discours collectif scolaire, ou dans les communautés discursives, pour reprendre une formule de Jaubert et Rebière, ceux qui restent à la lisière, voire en-deçà des verbalisations à visées proprement scolaires.

Si le discours horizontal rend difficile la perception des catégories du savoir, en les rendant implicites, le discours vertical, pour sa part, risque de les imposer en les réifiant; l'une des nécessités du discours pédagogique - peut-être la première - est donc d'instituer ce corps commun de catégories de pensées que représentent les disciplines, entendu comme une grammaire fiable mais évolutive du monde, et de considérer que c'est là une mission fondamentale de l'école. En second lieu, nous voudrions évoquer la nécessité de prendre en compte autrement qu'à partir d'activités de différenciation - souvent elles-mêmes inégalitaires - les différentes dispositions dont sont porteurs les élèves à l'école. C'est sans doute en intégrant dans le discours collectif, déjà bénéfique à certains d'entre eux, des formes d'explicitation, non pas seulement des modes de réalisation de la tâche, mais aussi des enjeux discursifs des activités scolaires, que l'on parviendra mieux à prendre en compte ces inégalités de départ, que l'école contribue parfois à aggraver. La question de l'explicitation des enjeux des activités scolaires entraine à sa suite celle de la stabilisation du savoir, fut-il en cours d'acquisition, fort complexe, puisqu'il s'agit de jalonner pour chacun un parcours d'apprentissage, et non seulement de faire le bilan d'une séance. Ces jalons peuvent prendre des formes différentes, orales comme écrites, dans le fil du dialogue, au tableau... ou, au-delà de l'échange, prendre place dans les cahiers, dans les affichages, être identifiés dans les ressources diverses dont dispose la classe ; mais dans tous les cas, ils doivent être perçus par les élèves en tant que tels, et être réguliers, voire systématisés. Il ne s'agit pas seulement de prévoir des temps d'institutionnalisation des séances, mais bien de penser la stabilisation des acquis selon des modalités variées tout au long de celles-ci, afin de prendre en compte les besoins des uns et des autres.

Enfin, nous considérons comme nécessaire de revisiter le statut du savoir et du langage à l'école, l'un et l'autre trop souvent réifiés, et de favoriser le développement, pour les enseignants, pour les élèves, et plus généralement pour l'institution scolaire dans son ensemble (corps d'inspection, conseillers pédagogiques, etc.), d'une conception problématologique du savoir, articulant mieux savoir et questionnement. De ce point de vue, nous considérons que les didactiques gagneront à s'affranchir de la dichotomie langage objet vs langage moyen d'apprentissage. C'est en effet cette conception problématisée du savoir qui nous semble pouvoir contribuer à dépasser une conception parfois trop aristotélicienne de la langue, en faveur d'une conception non statique, réflexive et dynamique du langage : à l'école, c'est tout autant l'absence de savoir qui empêche de questionner le monde, que l'absence de questionnement du monde qui empêche de s'approprier des savoirs. 


\section{RÉFÉRENCES BIBLIOGRAPHIQUES}

Altet, M. (1994). Note de synthèse. Comment interagissent enseignants et élèves en classe ? Revue française de pédagogie, 107, 123-139.

Authier-Revuz, J. (1984). Hétérogénéité(s) énonciative(s). Langages, 73, 98-111.

Authier-Revuz, J. (2003). Le Fait autonymique: langage, langue, discours. Quelques repères. In J. Authier-Revuz, M. Doury \& M. Reboul-Touré (Eds.), Parler des mots - Le fait autonymique en discours (pp. 67-96). Paris : Presses Sorbonne nouvelle.

Bakhtine, M. (1979/1984). Esthétique de la création verbale (trad. par A. Aucouturier). Paris : Gallimard.

Bautier, É. (2008). Socialisation cognitive et langagière et discours pédagogique : analyser le discours pédagogique pour comprendre les inégalités sociales à l'école. In D. Frandji \& P. Vitale (Éds.), L'actualité de Basil Bernstein, discours pédagogiques, pouvoirs (pp. 133-150). Rennes: Presses universitaires de Rennes.

Bautier, É., \& Rayou, P. (2009). Les inégalités d'apprentissage. Programmes, pratiques et malentendus scolaires. Paris : Presses universitaires de France.

Bautier, É., \& Rochex, J.-Y. (1997). Ces malentendus qui font les différences. In J.-P. Terrail (Éd.), La scolarisation en France, critique de l'état des lieux (pp. 105-122). Paris : La Dispute.

Bautier, E., \& Rochex, J.-Y. (2004). Activité conjointe ne signifie pas significations partagées. In C. Moro \& R. Rickenmann (Éds.), Situation éducative et significations (Raisons éducatives ; pp. 199-220). Bruxelles : De Boeck.

Bernstein, B. (2007). Pédagogie, contrôle symbolique et identité. Théorie, recherche, critique (trad. par G. Ramognino-Le Déroff \& P. Vitale). Laval : Les Presses de l'Université de Laval.

Bourdieu, P. (1967/2007). Systèmes d'enseignement et systèmes de pensée. In J. Deauvieau \& J.-P. Terrail (Éds.), Les sociologues, l'école et la transmission des savoirs (pp. 17-42). Paris : La Dispute.

Bres, J. (2005). Savoir de quoi on parle : dialogue, dialogal, dialogique ; dialogisme, polyphonie... In J. Bres, P.-P. Haillet, S. Mellet, H. Nolke \& L. Rosier (Éds.), Actes du colloque de Cerisy. Dialogisme et polyphonie. Approches linguistiques (pp. 47-61). Bruxelles: De Boeck-Duculot.

Bres, J., \& Rosier, L. (2008). Réfractions: polyphonie et dialogisme, deux exemples de reconfigurations théoriques dans les sciences du langage francophones. Slavica Occitania, 25, 238-251.

Bronckart, J.-P. (1987). Interaction, discours, significations. Langue française, 74, 29-50.

Charlot B., Bautier, É., \& Rochex, J.-Y. (1992). École et savoir dans les banlieues... et ailleurs. Paris : A. Colin.

Danon-Boileau, L. (1999). Affect, éprouvé, émotion, sentiment : notions terminologiques. Revue française de psychanalyse, LXIII, 9-12.

Danon-Boileau, L. (2005). L'affect et l'absence aux origines du langage. In J.-M Hombert (Ed.), Aux origines des langues et du langage (pp. 292-307). Paris : Fayard. 
Danon-Boileau, L. (2006). Éloge de l'approximation. In R. Delamotte-Legrand, C. Hudelot \& A. Salazar Orvig (Éds.), Dialogues, mouvements discursifs, significations. Hommage à Frédéric François (pp. 259-268). Paris : L'Harmattan.

Delarue-Breton, C. (2012). Discours scolaire et paradoxe. Louvain: Academia-L'Harmattan.

Delarue-Breton, C. (2016). Inégalité d'accès au savoir, ou inégalité d'accès au questionnement? De l'étude du dialogisme du discours scolaire à l'étude de l'activité dialogique des élèves et des étudiants. Note de synthèse pour le diplôme d'HDR. Repéré à https://hal-upec-mlv.archives-ouvertes.fr/tel-01500276

Fabre, M. (2007). Des savoirs scolaires sans problèmes et sans enjeux. La faute à qui ? Revue française de pédagogie, 161, 69-78.

François, F. (1990). Dialogues, jeux de langage et espace discursif chez l'enfant jeune et moins jeune. In F. François (Éd.), La communication inégale. Heurs et malheurs de l'interaction verbale (pp. 33-111). Neuchâtel: Delachaux et Niestlé.

Gomila, C. (2007). Le discours métalinguistique de la classe de lecture : comment les enseignants de cours préparatoires et leurs élèves qui apprennent à lire parlent du langage. Thèse de doctorat en sciences du langage, Université Paris 3.

Halté, J.-F. (1981). Pour changer l'écrire. Pratiques, 29, 23-46.

Jaubert, M., \& Rebière, M. (2002). Parler et débattre pour apprendre : comment caractériser un oral réflexif? In J.-C. Chabanne \& D. Bucheton (Éds.), Parler et écrire pour penser, apprendre et se construire (pp. 163-186). Paris : Presses universitaires de France.

Maingueneau, D. (1998). Analyser les textes de communication. Paris : Dunod.

Maingueneau D. (2004). Le discours littéraire. Paratopie et scène d'énonciation. Paris : Armand Colin.

Ministère de l'Éducation nationale, de l'Enseignement supérieur et de la Recherche [MENESR] (2015). Programmes d'enseignement du cycle des apprentissages fondamentaux (cycle 2), du cycle de consolidation (cycle 3) et du cycle des approfondissements (cycle 4). Bulletin officiel spécial $n^{\circ} 11$ du 26 novembre. Repéré à https://www.education.gouv.fr/cid95812/au-bospecial-du-26-novembre-2015-programmes-d-enseignement-de-l-ecoleelementaire-et-du-college.html

Ministère de l'Éducation nationale, \& Ministère de l'Enseignement supérieur et de la Recherche [MEN \& MESR] (2008). Horaires et programmes d'enseignement de l'école primaire. Bulletin officiel hors-série $n^{\circ} 3$ du 19 juin. Repéré à https://www.education.gouv.fr/bo/2008/hs3/default.htm

Normand, C. (1996). Émile Benveniste : quelle sémantique ? Lynx, 8, 221-240.

Sinclair, J., \& Coulthard, R. (1975). Towards an analysis of discourse. The English used by teachers and pupils. Oxford: Oxford University Press.

Schneuwly, B. (1998). Genres et types de discours : considérations psychologiques et ontogénétiques. In Y. Reuter (Éd.), Les interactions lecture-écriture (pp. 155-173). Berne : Peter Lang. 
Veyrunes, P., \& Saury, J. (2009). Stabilité et auto-organisation de l'activité collective en classe: exemple d'un cours dialogué à l'école primaire. Revue française de pédagogie, 169, 67-76.

Winnicott, D.W. (1971/1975). Jeu et réalité. L'espace potentiel (trad. par C. Monod \& J.-B. Pontalis). Paris : Gallimard.

\section{Notice biographique}

Catherine Delarue-Breton, agrégée de Grammaire, est Professeure des Universités en Sciences du langage à l'Université de Rouen-Normandie (École Supérieure du Professorat et de l'Éducation). Elle est membre du laboratoire DYLIS (Dynamique du langage in situ) et chercheure associée au laboratoire CIRCEFT-Escol (Centre Interdisciplinaire de Recherche Culture, Éducation, Formation, Travail). Ses travaux concernent notamment la description formelle du dialogue scolaire et l'appropriation des discours attendus, à l'oral et à l'écrit, en contexte scolaire et universitaire.

ADRESSE IRED, 7 rue Thomas Becket, F-76821 Mont Saint Aignan

COURRIEL catherine.delarue-breton@univ-rouen.fr 